\title{
The decay of Batchelor and Saffman rotating turbulence
}

\author{
Tomas Teitelbaum ${ }^{1}$ and Pablo D. Mininni ${ }^{1,2}$ \\ 1 Departamento de Física, Facultad de Ciencias Exactas y Naturales, \\ Universidad de Buenos Aires, \& IFIBA, CONICET, \\ Ciudad Universitaria, 1428 Buenos Aires, Argentina. \\ 2 NCAR, P.O. Box 3000, Boulder, Colorado 8030\%-3000, U.S.A.
}

(Dated: July 13, 2012)

\begin{abstract}
The decay rate of isotropic and homogeneous turbulence is known to be affected by the largescale spectrum of the initial perturbations, associated with at least two cannonical self-preserving solutions of the von Kármán-Howarth equation: the so-called Batchelor and Saffman spectra. The effect of long-range correlations in the decay of anisotropic flows is less clear, and recently it has been proposed that the decay rate of rotating turbulence may be independent of the large-scale spectrum of the initial perturbations. We analyze numerical simulations of freely decaying rotating turbulence with initial energy spectra $\sim k^{4}$ (Batchelor turbulence) and $\sim k^{2}$ (Saffman turbulence) and show that, while a self-similar decay cannot be identified for the total energy, the decay is indeed affected by long-range correlations. The decay of two-dimensional and three-dimensional modes follows distinct power laws in each case, which are consistent with predictions derived from the anisotropic von Kármán-Howarth equation, and with conservation of anisotropic integral quantities by the flow evolution.
\end{abstract}




\section{INTRODUCTION}

Turbulent flows subject to background rotation are an important problem in fluid mechanics as several systems are affected by rotation. Rotation affects the Earth atmosphere and the oceans at large scales, it is crucial in many engineering flows such as in turbomachinery, and is also important in many astrophysical systems such as stellar convective regions and gaseous planets atmospheres. As many applications arise in nature and technology, it becomes important to understand detailed properties of these flows.

In particular, a long-lasting problem in fluid mechanics is that of the decay of turbulent fluctuations. The rate of energy decay in turbulent flows is known to be sensitive to initial conditions, and no single universal solution to the decay appears to exist to which

all flows converge asymptotically for infinite Reynolds number [1]. Considering isotropic and homogeneous turbulence at high Reynolds number, there are two well known canonical cases as far as the energy spectrum at large scales is concerned. Provided the cubic velocity correlation tensor $\left\langle u_{i}(\mathbf{x}) u_{j}(\mathbf{x}) u_{k}(\mathbf{x}+\mathbf{r})\right\rangle$ decays sufficiently fast for large $r$, these cases correspond to the so-called Saffman spectrum for which $E(k \rightarrow 0) \sim L k^{2}$, and to the so-called Batchelor spectrum where $E(k \rightarrow 0) \sim I k^{4}$. Which of these spectra is observed depends on the initial conditions, and other non-canonical cases may arise if the above condition on the decay of the correlation tensor does not hold (see, e.g., [2]). The quantities $L$ and $I$ are approximately conserved integrals for fully developed, freely decaying turbulence in each case ( $I$ is often called the Loytsiansky integral), and have been used to determine the decay rate of the total energy (see [3] 6] for examples). The quasi-conservation in each case is associated with self-preserving solutions of the von Kármán-Howarth equations, and can also be interpreted in terms of conservation of linear and angular momentum. While constancy of $L$ is a consequence of linear momentum conservation [5], constancy of $I$ is a consequence of the conservation of angular momentum [7, 8].

The presence of background rotation breaks down isotropy as a preferred direction arises along the axis of rotation (for detailed studies of rotating turbulence, see [9]11]). Flows subjected to rotation develop anisotropies which have been shown to impact dramatically on the decay of energy, and there are different and sometimes conflicting results in the literature regarding the decay laws followed by the energy in rotating flows. Moreover, theoretical arguments based on the isotropic von Kármán-Howarth equations are not valid 
anymore, and must be extended to include the effect of rotation. The integrals $I$ and $L$ become tensors, and for axisymmetric flows some of the diagonal components of these tensors can be expected to replace $I$ and $L$ as approximately conserved quantities. Extensions of these arguments to anisotropic cases such as conducting flows with an imposed magnetic field, rotating, or stratified flows, have been recently derived in Refs. [7, 12, 13].

The development of anisotropies in rotating flows have been reported both in experiments and simulations. Experiments of freely decaying rotating turbulence show a reduction of the energy transfer, evidenced by a decrease in the energy decay rate [14]. An anisotropic energy flux with a trend towards quasi-two-dimensionalization has been recently reported [15], and is also evidenced, e.g., by an increase in the correlation lengths along the axis of rotation [16, 17]. Regarding the energy decay, experimental results for non-rotating grid turbulence with integral scales smaller than the size of the vessel [18] show an energy decay $E \sim t^{-1.1}$ compatible with the theoretical result $E \sim t^{-6 / 5}$ expected for large scale initial conditions with Saffman spectrum $\sim k^{2}$. In [19], a transition from a $E \sim t^{-6 / 5}$ decay to a decay law closer to $\sim t^{-3 / 5}$ is reported once rotation starts affecting the flow dynamics, together with a cyclone-anticyclone asymmetry evidenced by positive skewness which grows with rotation rate. The decay for bounded flows, in which initial integral scales are close to the size of the vessel, seems to change from $E \sim t^{-2}$ to $\sim t^{-1}$ when rotation is present [18].

Numerical simulations and models also show a slow down of the energy decay for many different initial conditions [11, 20 24], together with the trend towards two-dimensionalization [22, 25], and the higher cyclonic-over-anticyclonic activity 26, 27]. The first theoretical study of the decay of rotating turbulence was reported in [28], and considered the decrease of the energy transfer in the presence of rotation to explain the observed slow down in the decay, and to predict decay rates for the energy. The exponents reported were consistent with those obtained in simulations in [20], and in the experiments in [18]. Also, a very close decay law $E \sim t^{-0.8}$ was found in [11] for a wide range of Rossby numbers (from very small to moderate values), when solving equations for inertial wave turbulence and for an Eddy Damped Quasi-Normal Markovian model (EDQNM3).

Later, in [25] a distinction was introduced between the energy contained in modes with $k_{z}=0\left(E_{2 D}\right.$, corresponding to two-dimensional, "slow" or "vortical" modes) and modes with $k_{z} \neq 0$ ( $E_{3 D}$, corresponding to three-dimensional, "fast", or "wave" modes). The authors carried a comprehensive study of the transfer of energy from 3D to 2D modes, for a wide 
range of Rossby numbers, and found a non-monotonic behavior for the decay of energy for large, intermediate, and small Rossby numbers. They focused on the intermediate Rossby range, where the maximal energy transfer between 3D and 2D modes occurs (see also [29]). In this range, they reported an initial growth of $E_{2 D}$ as soon as the decay begins, that eventually results in a cross-over with the monotonously decaying $E_{3 D}$. Reference [30] also reported different behavior in the time evolution of $E_{2 D}$ and $E_{3 D}$, but considered only the decay of the total energy $E=E_{2 D}+E_{3 D}$ proposing a decay that depends on the amount of background rotation $E \sim t^{-\gamma(\Omega)}$. The dependence of $\gamma$ on the rotation rate was derived using phenomenological arguments resulting $\gamma \sim \Omega^{-1}$.

This variety of solutions naturally led to parametric studies of the initial condition space in numerical simulations. For non-helical rotating flows with initial integral scale close to the size of the domain [24], a decay $E \sim t^{-1}$ was found in simulations, in agreement with the experimental results [18]. For flows with initial integral scale sufficiently smaller than the domain size, the case of initial conditions with energy spectra $\sim k^{4}$ was studied in detail in Refs. [26, 27], where different decay rates were reported for the two-dimensional and threedimensional modes. While $E_{3 D}$ was found to decay as in the case of isotropic turbulence, a slower decay was found for $E_{2 D}$ in the intermediate-Rossby range as defined in [25]. The decay of two-dimensional modes can be correctly explained if only a few components of the Loytsiansky tensor are considered (here referred in short as $I_{\perp}$ and $I_{\|}$based on the symmetries of the flow), and it was shown that $I_{\perp}$ remains approximately constant during the decay [27]. Finally, it was also found that the decay is influenced by the amount of total helicity present in the initial conditions, and by the initial degree of anisotropy in the flow.

Recently, the anisotropic von Kármán-Howarth equation was used to consider the decay of a rotating turbulent flow with initial conditions following a Saffman spectrum [12]. The approximately conserved integral $L$ in the isotropic and homogeneous case, was generalized in the axisymmetric case to two integrals $L_{\perp} \propto L_{x x}=L_{y y}$ and $L_{\|}=L_{z z}$. Assuming that both $L_{\perp}$ and $L_{\|}$are simultaneously conserved (or $I_{\perp}$ and $I_{\|}$for the case of Batchelor initial spectrum), and using the empirical result that characteristic lengthscales of the flow in the direction parallel to the rotation axis grow linearly with time [12], the author concludes that regardless of the initial spectrum being $\sim k^{2}$ or $\sim k^{4}$, both cases should decay with the same $E \sim t^{-1}$ law, thus implying that it is not possible to set apart $\sim k^{2}$ from $\sim k^{4}$ turbulence by measuring the energy decay exponent alone. 
In this paper we analyze whether the decay of rotating flows is affected by long-range correlations, studying the energy decay rate for flows with $\sim k^{2}$ and $\sim k^{4}$ initial conditions. We first present a brief theoretical discussion of conserved integral quantities for rotating turbulence using a von Kármán-Howarth equation which includes a Coriolis term due to rotation. We extend the previously derived results for Saffman turbulence [12], considering Batchelor spectrum. The results corroborate the phenomenological arguments used in [27] to explain the decay laws found numerically for energy spectra $k^{4}$. In the second part of the paper we use numerical simulations to analyze the decay of rotating Saffman and Batchelor turbulence in periodic domains. We focus on the regime of intermediate Rossby numbers (initial $R_{o} \approx 0.1$ [25]), and therefore we will not attempt a study of the dependence of the decay with the rotation rate. To the best of our knowledge, the free decay of energy for $\sim k^{2}$ initial conditions has not yet been numerically studied for rotating turbulence, and the resulting decay laws seem to be relevant for experiments. We show that the decay of energy in two-dimensional and three-dimensional modes follows distinct power laws for the cases of Batchelor and Saffman turbulence. The decay laws are consistent with the predictions derived from the anisotropic von Kármán-Howarth equation, and with the approximate constancy of either $L_{\perp}$ or $I_{\perp}$ as the flow decays.

\section{VON KÁRMÁN-HOWARTH EQUATIONS AND SELF-SIMILAR DECAY}

The dynamics of an incompressible fluid subjected to background rotation is described by the Navier-Stokes equation with the addition of the Coriolis acceleration,

$$
\partial_{t} \mathbf{u}+\boldsymbol{\omega} \times \mathbf{u}+2 \boldsymbol{\Omega} \times \mathbf{u}=-\nabla \mathcal{P}+\nu \nabla^{2} \mathbf{u}
$$

together with the incompressibility condition,

$$
\nabla \cdot \mathbf{u}=0
$$

Here, $\mathbf{u}$ is the velocity field, $\boldsymbol{\omega}=\nabla \times \mathbf{u}$ is the vorticity, the centrifugal acceleration is absorbed in the total pressure per unit of mass $\mathcal{P}$, and $\nu$ is the kinematic viscosity. We assume uniform density and the rotation axis in the $z$ direction so $\Omega=\Omega \hat{z}$, with $\Omega$ the rotation frequency.

We briefly present the derivation of the von Kármán-Howarth equation including the Coriolis term in order to find invariant quantities during the self-similar energy decay in 
rotating flows. To this end, we write the Navier-Stokes equation in a rotating frame in index notation, and evaluated at two points $\mathbf{x}$ and $\mathbf{x}^{\prime}=\mathbf{x}+\mathbf{r}$,

$$
\frac{\partial u_{i}}{\partial t}=-\frac{\partial}{\partial x_{k}}\left(u_{i} u_{k}\right)-\frac{\partial p}{\partial x_{i}}+\nu \nabla^{2} u_{i}-2 \epsilon_{i m n} \Omega_{m} u_{n}
$$

and

$$
\frac{\partial u_{j}^{\prime}}{\partial t}=-\frac{\partial}{\partial x_{k}^{\prime}}\left(u_{j}^{\prime} u_{k}^{\prime}\right)-\frac{\partial p^{\prime}}{\partial x_{j}^{\prime}}+\nu \nabla^{\prime 2} u_{j}^{\prime}-2 \epsilon_{j m n} \Omega_{m} u_{n}^{\prime},
$$

where $\mathbf{u}^{\prime}=\mathbf{u}\left(\mathbf{x}^{\prime}\right), \nabla^{\prime 2}$ denotes Laplacian with respect to the $\mathbf{x}^{\prime}$ coordinate, and $\epsilon$ is the Levi-Civita symbol. Multiplying Eq. (3) by $u_{j}^{\prime}$, Eq. (44) by $u_{i}$, summing and averaging, we get the equation for the time evolution of the two-point velocity correlation tensor,

$$
\begin{aligned}
& \frac{\partial}{\partial t}\left\langle u_{i} u_{j}^{\prime}\right\rangle=-\left(\frac{\partial}{\partial x_{k}}\left\langle u_{j}^{\prime} u_{i} u_{k}\right\rangle+\frac{\partial}{\partial x_{k}^{\prime}}\left\langle u_{i} u_{j}^{\prime} u_{k}^{\prime}\right\rangle\right) \\
& -\left(\frac{\partial}{\partial x_{i}}\left\langle u_{j}^{\prime} p\right\rangle+\frac{\partial}{\partial x_{j}^{\prime}}\left\langle u_{i} p^{\prime}\right\rangle\right)+\nu\left(\nabla^{2}\left\langle u_{j}^{\prime} u_{i}\right\rangle+\right. \\
& \left.+\nabla^{\prime 2}\left\langle u_{i} u_{j}^{\prime}\right\rangle\right)-2 \Omega_{m}\left\langle\epsilon_{i m n} u_{n} u_{j}^{\prime}+\epsilon_{j m n} u_{n}^{\prime} u_{i}\right\rangle .
\end{aligned}
$$

If $\Omega=0$, and assuming the cubic velocity correlation tensor $\left\langle u_{i} u_{j} u_{k}^{\prime}\right\rangle$ and the pressurevelocity correlation $\left\langle u_{i} p^{\prime}\right\rangle$ decay fast enough with $r$ when $r \rightarrow \infty$, two possible integral conserved quantities can be obtained from Eq. (5). When the terms on the r.h.s. of the equation go to zero as $\mathcal{O}\left(r^{-2}\right)$, then it follows from the time derivative on the 1.h.s. that

$$
L=\int\left\langle\mathbf{u} \cdot \mathbf{u}^{\prime}\right\rangle d \mathbf{r}
$$

is conserved. This is know as the Saffman integral [5]. Multiplying Eq. (50) by $r^{2}$, another possible invariant is obtained when the terms on the l.h.s. are $\mathcal{O}\left(r^{-4}\right)$, namely

$$
I=-\int r^{2}\left\langle\mathbf{u} \cdot \mathbf{u}^{\prime}\right\rangle d \mathbf{r}
$$

which is known as the Loitsyanski integral and whether it actually remains constant or not in decaying isotropic and homogeneous turbulence has been a matter of debate [31]. As the energy spectrum for small values of $k$ can be expanded as

$$
E(k \rightarrow 0) \sim L k^{2}+I k^{4}+\ldots,
$$

the integrals $L$ or $I$ are then expected to be quasi-invariants during the decay for initial largescale energy spectra of the form $\sim k^{2}$ (Saffman spectrum) [5], 6] or $\sim k^{4}$ (Batchelor spectrum) 
44] respectively. Using these quasi-conserved integrals, the energy balance equation $d E / d t \sim$ $-E^{3 / 2} / l$ can be closed (as either $l^{3} u^{2}$ or $l^{5} u^{2}$ are constant, with $l$ the energy-containing scale), and different decay laws arise for each scaling of the initial spectra,

$$
E(t) \sim \begin{cases}t^{-6 / 5} & \text { if } E(k, t=0) \sim k^{2} \\ t^{-10 / 7} & \text { if } E(k, t=0) \sim k^{4}\end{cases}
$$

These decay laws has been observed in numerical as well as in experimental studies (see, e.g., [3, 5, 6, 18, 19, 27, 32])

These isotropic integrals have been also assumed to remain constant during the decay of rotating turbulence with $\sim k^{2}$ and $\sim k^{4}$ large-scale energy spectra, to predict energy decay laws [28]. In the presence of rotation, the energy per unit of time (flux) transferred towards smaller scales is reduced as $\Omega$ is increased. A phenomenological expression consistent with the observations is to assume that the flux is reduced by the ratio of the turnover time $\tau \sim l / E^{1 / 2}$ to the wave time $(2 \Omega)^{-1}[22$, 33 35], resulting in [28]

$$
\frac{d E}{d t} \sim-\frac{E^{2}}{\Omega l^{2}}
$$

Further assuming $I$ or $L$ are conserved results in the following decays,

$$
E(t) \sim \begin{cases}t^{-3 / 5} & \text { if } E(k, t=0) \sim k^{2} \\ t^{-5 / 7} & \text { if } E(k, t=0) \sim k^{4}\end{cases}
$$

Although these power laws are close to the behavior found in experiments and simulations, as mentioned in the introduction a better agreement with observations can be obtained when the arguments are extended to consider the effect of anisotropy in the flow.

In the anisotropic case, and assuming absence of long-range correlations, only the final term in Eq. (5) can contribute to the rate of change of integrals of the type

$$
I_{i j m n}=\int r_{m} r_{n}\left\langle u_{i} u_{j}^{\prime}\right\rangle d \mathbf{r}
$$

for the Batchelor case, or

$$
L_{i j}=\int\left\langle u_{i} u_{j}^{\prime}\right\rangle d \mathbf{r}
$$

for Saffman turbulence. It is important to point out here that it is not trivial that in this case long-range correlations should vanish as in the isotropic case (specially for the pressure velocity correlations, see [11] where these terms are computed using the Fourier transform 
of Eq. (5) and the Poisson equation for the pressure fluctuations). Thus, the validity of the assumptions can only be verified a posteriori from the results obtained from the numerical simulations.

Assuming long-range correlations vanish sufficiently fast at large-scales, the problem is that of finding what components of the tensors in Eqs. (12) and (13) are still conserved in the presence of rotation. We derive here the Batchelor case $\left(I_{i j m n}\right)$ under the same assumptions for the decay of velocity correlations with $r$ as in the isotropic case, multiplying Eq. (5) by $r_{m} r_{n}$ and integrating to obtain

$$
\begin{aligned}
& \frac{\partial}{\partial t} \int r_{m} r_{n}\left\langle u_{i} u_{j}^{\prime}\right\rangle d \mathbf{r}= \\
& -2 \Omega_{l} \int r_{m} r_{n}\left(\epsilon_{i l k}\left\langle u_{k} u_{j}^{\prime}\right\rangle+\epsilon_{j l k}\left\langle u_{k}^{\prime} u_{i}\right\rangle\right) d \mathbf{r} .
\end{aligned}
$$

Let's assume $\Omega_{l}=\Omega_{z} \delta_{l z}$, so

$$
\begin{aligned}
& \frac{\partial}{\partial t} \int r_{m} r_{n}\left\langle u_{i} u_{j}^{\prime}\right\rangle d \mathbf{r}= \\
& -2 \Omega_{z} \int r_{m} r_{n}\left(\epsilon_{i z k}\left\langle u_{k} u_{j}^{\prime}\right\rangle+\epsilon_{j z k}\left\langle u_{k}^{\prime} u_{i}\right\rangle\right) d \mathbf{r} .
\end{aligned}
$$

As the flow has axisymmetry, we are interested in the diagonal components of the tensor. For $j=i$,

$$
\begin{aligned}
& \Omega_{z} \epsilon_{i z k}\left\langle u_{k} u_{i}^{\prime}\right\rangle=\Omega_{z}\left\langle u_{x} u_{y}^{\prime}-u_{y} u_{x}^{\prime}\right\rangle, \\
& \Omega_{z} \epsilon_{j z k}\left\langle u_{k}^{\prime} u_{j}\right\rangle=\Omega_{z}\left\langle u_{x}^{\prime} u_{y}-u_{y}^{\prime} u_{x}\right\rangle,
\end{aligned}
$$

where all terms with $i=z$ or $j=z$ vanish. For $m=n$, introducing $\mathbf{r}_{\perp}=\left(r_{x}, r_{y}, 0\right)$ and $\mathbf{u}_{\perp}=\left(u_{x}, u_{y}, 0\right)$ we get

$$
\begin{aligned}
& \frac{\partial}{\partial t} \int r_{\perp}^{2}\left\langle u_{\perp} u_{\perp}^{\prime}\right\rangle d \mathbf{r}= \\
& -2 \int r_{\perp}^{2} \Omega_{z}\left[\left\langle u_{x} u_{y}^{\prime}-u_{y} u_{x}^{\prime}\right\rangle-\left\langle u_{x} u_{y}^{\prime}-u_{y} u_{x}^{\prime}\right\rangle\right] d \mathbf{r}
\end{aligned}
$$

so the terms on the r.h.s. of the equation cancel and

$$
\frac{\partial}{\partial t} \int r_{\perp}^{2}\left\langle\mathbf{u}_{\perp} \mathbf{u}_{\perp}^{\prime}\right\rangle d \mathbf{r}=0,
$$

or equivalently,

$$
I_{\perp}=\int r_{\perp}^{2}\left\langle\mathbf{u}_{\perp} \mathbf{u}_{\perp}^{\prime}\right\rangle d \mathbf{r}=\text { constant }
$$


The case of Saffman turbulence was considered in [12]. The relevant components of the tensor $L_{i j}$ for axisymmetric flows are $L_{x x}=L_{y y}$ and $L_{z z}$. Similar arguments as the ones described above lead, for the $x x$ and $y y$-components of the von Kármán-Howarth equation, to the conservation of the integral

$$
L_{\perp}=\int\left\langle\mathbf{u}_{\perp} \mathbf{u}_{\perp}^{\prime}\right\rangle d \mathbf{r}=\text { constant. }
$$

Note that both $I_{\perp}$ and $L_{\perp}$ resemble integrals that arise in the context of the decay of $2 D$ turbulence [36]. This is to be expected as the spectrum of an axisymmetric flow can be expanded, for the modes with $k_{\|}=k_{z}=0$, as

$$
E\left(k_{\perp} \rightarrow 0, k_{\|}=0\right) \approx L_{\perp} k_{\perp}+I_{\perp} k_{\perp}^{3}+\ldots
$$

which is (except for differences in the dimensions) also the expansion of a 2D energy spectra. The resemblance can be expected as rotating flows tend to become quasi-2D, concentrating most of the energy in the slow modes with $k_{\|}=0$.

For axisymmetric turbulence and a Batchelor initial spectrum $\sim k^{4}$ at large scales, Eq. (20) leads to

$$
I_{\perp} \sim l_{\perp}^{4} l_{\|} u_{\perp}^{2} \approx \text { constant }
$$

where $l_{\perp}$ and $l_{\|}$are characteristic (energy-containing) scales in the perpendicular and parallel direction respectively. Assuming 2D and 3D modes are only weakly coupled in rotating turbulence, from Eq. (10) we can write an equation for the decay of the energy in 2D modes

$$
\frac{d E_{2 D}}{d t} \sim-\frac{E_{2 D}^{2}}{\Omega I_{\perp}^{2}},
$$

and from Eq. (23)

$$
\frac{d E_{2 D}}{d t} \sim-\frac{E_{2 D}^{5 / 2} l_{\|}^{1 / 2}}{I_{\perp}^{1 / 2} \Omega} .
$$

Assuming $E_{2 D} \sim t^{\gamma}$ it follows that [27]

$$
E_{2 D} \sim t^{-2 / 3}
$$

if $l_{\|}$remains constant (which is reasonable as $2 \mathrm{D}$ modes have no dependence on the direction parallel to rotation, and therefore $l_{\|}=l_{0}$ is the vertical size of the box).

For $\mathrm{a} \sim k^{2}$ initial spectra,

$$
L_{\perp} \sim l_{\perp}^{2} l_{\|} u_{\perp}^{2} \approx \text { constant }
$$


and replacing in Eq. (24) leads to

$$
\frac{d E_{2 D}}{d t} \sim-\frac{E_{2 D}^{3} l_{\|}}{L_{\perp} \Omega}
$$

and a decay

$$
E_{2 D} \sim t^{-1 / 2}
$$

The results for the decay of energy in 2D modes in rotating turbulence can be summarized as follows,

$$
E_{2 D}(t) \sim \begin{cases}t^{-1 / 2} & \text { if } E(k, t=0) \sim k^{2} \\ t^{-2 / 3} & \text { if } E(k, t=0) \sim k^{4} .\end{cases}
$$

It is worth mentioning that the decay of rotating turbulence with $\sim k^{2}$ spectra is discussed in detail in [12], where the constancy of $L_{\perp}$ is obtained from the Kármán-Howarth equation. In that case, the author also considers the von Kármán-Howarth equation for the $z z$-component of the two-point correlation tensor, and assuming cubic correlations decay fast enough also for that component, obtains constancy of $L_{\|} \sim u_{\|}^{2} l_{\perp}^{2} l_{\|}$as well as of $L_{\perp}$. The constancy of these two quantities, together with the empirical result $l_{\|}=l_{0}(1+\kappa \Omega t)$ (where $\kappa$ is a constant of order one [16, 20]) leads to a decay $E_{2 D}(t) \sim t^{-1}$. A similar argument for Batchelor $\sim k^{4}$ turbulence leads to the same decay, which would result in an impossibility to distinguish between $\sim k^{2}$ and $\sim k^{4}$ turbulence by measuring the energy decay exponent alone.

Finally, it is interesting to point out that constancy of $I_{\perp}$ and $L_{\perp}$ can be respectively associated with conservation of the z-component of angular and linear momentum (see [7, 12]). In fact, the mean squared angular momentum $H_{z}$ averaged over a cylinder-shaped volume $V$ is

$$
\left\langle H_{z}^{2}\right\rangle=2 \pi V l_{\|} I_{\perp}
$$

while the linear momentum $P_{z}$ is

$$
\left\langle L_{z}^{2}\right\rangle=2 \pi V l_{\|} L_{\perp}
$$

\section{NUMERICAL SIMULATIONS}

In the following we resort to numerical simulations to verify the validity of the assumptions and predictions discussed in the previous section. As a well resolved initial spectrum $\sim k^{2}$ or 
$\sim k^{4}$ is needed to observe constancy of $I$ or $L$ in simulations of isotropic and homogeneous

turbulence (see, e.g., [3]), we use large-eddy simulations (LES) in periodic boxes to be able to have initial energy containing wavenumber $k_{0} \approx 40$ with large Reynolds numbers at a reasonable computing cost.

Before proceeding, we must caution the reader on how the simulations in periodic boxes should be interpreted. Flows in periodic boxes are sometimes considered as describing a homogeneous flow in an infinitely periodic domain. In this work, we will interpret instead a flow in a periodic box as artificially confined. Indeed, as was shown in [27], when the integral scale of the flow is close to the domain size, the eddies cannot become larger and the energy decay laws obtained are those of confined flows. The confinement is artificial in the sense that the flows have no Ekman layers, as the domain has no rigid walls. Moreover, the confinement also results in a discrete set of wavenumbers and selects a discrete set of inertial waves which are normal modes of the domain, unlike homogeneous rotating flows in infinite domains which have continuous wavenumbers (see, e.g., [11, 37]). Note that due to the discretization of wavenumbers in the former case, the existence and number of exact resonances depend on the domain size [37] (as this is aggravated when boxes with large aspect ratio and lower resolution in the vertical direction are used, we restrict our study to boxes with aspect ratio of unity).

While these are unavoidable and inherent properties of the simulations in periodic domains, the choice of a relatively large initial energy containing wavenumber $k_{0} \approx 40$ ensures that as the equations are advanced in time the energy containing scale of the flow will remain smaller than the box size, and that a large fraction of wavenumbers will be available at large-scales for the system to evolve.

\section{A. Equations and model}

Equations (11) and (21) are solved numerically using a dynamical subgrid-scale spectral LES model of rotating turbulence in which only the large scales are explicitly resolved. The statistical effect of unresolved scales on the scales larger than a cut-off wave number $\left(k_{c}=N / 2\right)$ are modeled with an eddy-viscosity and eddy-noise that are obtained after solving in each time step the eddy damped quasi-normal Markovian (EDQNM) equations for the spectrum of the unresolved scales. Details of the LES can be found in Ref. [38], 
and in Refs. [39, 40] for its extension to the rotating case. A validation of the LES against direct numerical simulations (DNS) for the case of freely decaying rotating turbulence can be found in [27].

The simulation domain is a $3 \mathrm{D}$ periodic box of length $2 \pi$ with spatial resolution of $256^{3}$ grid points (resulting in $k_{c}=128$ ). A parallelized pseudo-spectral method without de-aliasing is used to solve spatial derivatives, and an explicit second-order Runge-Kutta method is used to evolve in time [41].

\section{B. Initial conditions}

In 27] it was observed that the energy decay is sensitive to the initial anisotropy of the flow (in particular, to the initial amount of energy in 2D modes). To enforce spectral and variance isotropy of the initial conditions, we generate at $t=0$ a random velocity field using the Craya-Herring decomposition [42, 43] which associates to each wave vector $\mathbf{k}$ an orthonormal frame with axes dependant on $\mathbf{k}$ as

$$
\mathbf{i}(\mathbf{k})=\frac{\mathbf{k} \times \alpha}{|\mathbf{k} \times \alpha|}, \quad \mathbf{j}(\mathbf{k})=\frac{\mathbf{k} \times \mathbf{i}}{|\mathbf{k}|}, \quad \frac{\mathbf{k}}{k}
$$

$\alpha$ is an arbitrary but fixed unit vector which we choose to be parallel to the rotation axis, $\alpha=(0,0,1)$. This representation has been widely used to study turbulence (see, e.g., [44, 45]). Random initial conditions are created through a superposition of harmonic modes with random phases $\phi(\mathbf{k}), \phi^{\prime}(\mathbf{k})$ projected over the Craya reference system for each $\mathbf{k}$,

$$
\mathbf{u}(\mathbf{k})=e^{i \phi} \mathbf{i}+e^{i \phi^{\prime}} \mathbf{j}
$$

Note that due to the geometry of the Craya-Herring decomposition there is no projection of $\mathbf{u}$ into $\mathbf{k}$ as the incompressibility condition stands for $\mathbf{u}(\mathbf{k}) \perp \mathbf{k}$.

As we are interested in the decay laws followed by flows with a particular initial spectrum (either Batchelor's or Saffman's), we can further control the shape of the initial isotropic spectrum by multiplying each mode $\mathbf{u}(\mathbf{k})$ in Fourier space with $0<k \leq k_{0}$ by an amplitude $C k^{\beta}$, where $\mathrm{C}$ is a constant to get initial r.m.s. velocity $U \approx 1$, and $\beta$ is a parameter used to adjust the power law in $E(k)$. For $k>k_{0}$, the spectrum is continued by an exponential decay up to $k_{c}$ (the maximum wavenumber explicitly resolved in the simulations). 


\section{Spectra}

In order to characterize the simulations we consider isotropic as well as anisotropic spectra. The latter is useful to analyze rotating flows in which anisotropies grow with time.

In the simulations, the isotropic spectrum is defined by averaging in Fourier space over spherical shells,

$$
E(k, t)=\frac{1}{2} \sum_{k \leq|\mathbf{k}|<k+1} \mathbf{u}^{*}(\mathbf{k}, t) \cdot \mathbf{u}(\mathbf{k}, t),
$$

where the star denotes complex conjugate. For the anisotropic spectrum the components of $\mathbf{u}(\mathbf{k})$ are integrated around the axis of rotation to obtain a spectrum that depends only on $k_{\perp}$,

$$
E\left(k_{\perp}\right)=\frac{1}{2} \sum_{k_{\perp} \leq\left|\mathbf{k}_{\perp}\right|<k_{\perp}+1} \mathbf{u}^{*}(\mathbf{k}, t) \cdot \mathbf{u}(\mathbf{k}, t) .
$$

From now on we will reference these two spectra simply as $E(k)$ and $E\left(k_{\perp}\right)$ respectively.

More detailed information on the energy spectral distribution of anisotropic flows can be obtained from the axisymmetric energy spectrum $e\left(k_{\|}, k_{\perp}\right)$ (see, e.g., [10, 45, 46]). This spectrum is obtained after integrating the three-dimensional energy spectrum around the axis of rotation to obtain a spectrum that depends only on $k_{\|}$and $k_{\perp}$, and that relates to $E\left(k_{\perp}\right)$ as follows:

$$
E\left(k_{\perp}\right)=\sum_{k_{\|}} e\left(k_{\|}, k_{\perp}\right) .
$$

Finally, the spectrum of the initial conditions is such that the Saffman or Batchelor power laws $\sim k^{2}$ or $\sim k^{4}$ are imposed in $E(k)$. The relationship between the power law in $E(k)$ and of that of $E\left(k_{\perp}\right)$ can be derived as follows. Equation (35) in the continuous limit can be expressed as

$$
E(k, t)=\frac{1}{2} \int \hat{\Phi}_{i i}(k, t) k^{2} d \Omega_{k}
$$

where $\hat{\Phi}_{i j}$ is the spectral tensor, i.e., the Fourier transform of the second order velocity correlation function $\Phi_{i j}\left\langle u_{i}(\mathbf{x}, t) u_{j}(\mathbf{x}+\mathbf{r}, t)\right\rangle$, and where $\Omega_{k}$ is the solid angle in Fourier

space. Per virtue of Eq. (38), if $E(k, 0) \sim k^{\sigma}$ then $\hat{\Phi}_{i i} \sim k^{\sigma-2}$. For axisymmetric flows we can write the energy spectrum $E\left(k_{\perp}\right)$ integrating in complex space over cylindrical shells,

$$
E\left(k_{\perp}, t\right)=\frac{1}{2} \int \hat{\Phi}_{i i}\left(k_{\perp}, t\right) k_{\perp} d \phi_{k} d k_{z} .
$$


TABLE I: Parameters used in the simulations: number of linear grid points $N$,kinematic viscosity $\nu$, rotation frequency $\Omega$, Reynolds number $R e$, Taylor Reynolds number $R e_{\lambda}$, Rossby number $R o$, micro-Rossby number $R o^{\omega}$, and a brief description of the initial energy spectrum $E(k)$ : the power law followed at large-scales and the range of scales where this power law is satisfied. Values of Re, $R e_{\lambda}, R o$, and $R o_{\lambda}$ are given at $t=0$, and at $t=20$ between parentheses.

\begin{tabular}{lcccccccc}
\hline \hline Run & $N$ & $\nu$ & $\Omega$ & $R e$ & $R e_{\lambda}$ & $R o$ & $R o^{\omega}$ & Initial $E(k)$ \\
\hline A & 256 & $1.3 \times 10^{-4}$ & 0 & $1580(330)$ & $780(100)$ & $\infty(\infty)$ & $\infty(\infty)$ & $k^{2}(1 \leq k \leq 40)$ \\
B & 256 & $1.3 \times 10^{-4}$ & 33 & $1580(1090)$ & $780(520)$ & $0.15(0.004)$ & $0.9(0.024)$ & $k^{2}(1 \leq k \leq 40)$ \\
C & 256 & $1.3 \times 10^{-4}$ & 0 & $1280(150)$ & $730(60)$ & $\infty(\infty)$ & $\infty(\infty)$ & $k^{4}(1 \leq k \leq 40)$ \\
D & 256 & $1.3 \times 10^{-4}$ & 33 & $1280(360)$ & $730(180)$ & $0.18(0.002)$ & $1.0(0.015)$ & $k^{4}(1 \leq k \leq 40)$ \\
\hline \hline
\end{tabular}

If $E(k, 0) \sim k^{\sigma}$ then,

$$
\begin{array}{r}
E\left(k_{\perp}, 0\right)=\frac{1}{2} \int k^{\sigma-2} k_{\perp} d \phi_{k} d k_{z}= \\
\frac{1}{2} \int\left(k_{\perp}^{2}+k_{z}^{2}\right)^{\frac{\sigma-2}{2}} k_{\perp} d \phi_{k} d k_{z}
\end{array}
$$

When most of the energy is in the $k_{z}=0$ plane then, from Eq. (40)

$$
E\left(k_{\perp}, 0\right) \sim \frac{1}{l_{\|}} \int k_{\perp}^{\sigma-1} d \phi_{k} .
$$

Even in the case in which the energy is not concentrated in the $k_{z}=0$ plane (as in the isotropic initial conditions), the dependence of $E\left(k_{\perp}\right)$ will be $\sim k_{\perp}^{\sigma-1}$ as it follows directly from Eq. (40). To summarize,

$$
E(k) \sim k^{\sigma} \Rightarrow E\left(k_{\perp}\right) \sim k_{\perp}^{\sigma-1}
$$

It is important to note that this simple relation between the isotropic $E(k)$ and the anisotropic $E\left(k_{\perp}\right)$ spectra only holds for the isotropic initial conditions. However, the relation will be useful to understand the power laws observed at large scales in the following section. As an example, when analyzing rotating turbulence with an initial isotropic spectrum $E(k) \sim k^{2}$, we will be facing the case with initial $E\left(k_{\perp}\right) \sim k_{\perp}$. Note the power laws $E\left(k_{\perp}\right) \sim k_{\perp}$ and $E\left(k_{\perp}\right) \sim k_{\perp}^{3}$, resulting respectively for Saffman and Batchelor isotropic initial conditions, are the ones that arise in the expansion of the axisymmetric energy spectrum for $k_{\|}=0$ and $k_{\perp} \rightarrow 0$ in Eq. (22) . 


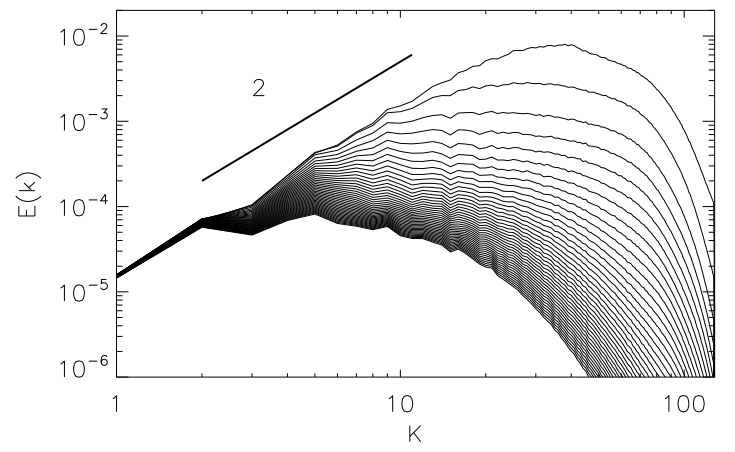

FIG. 1: Evolution of the isotropic energy spectrum $E(k)$ for run A from $t=0$ to $t=20$ with time increments $\Delta t=0.5$. Note how the initial $E \sim k^{2}$ spectrum is preserved over time at the larges scales (smaller wave numbers).

\section{NUMERICAL RESULTS}

We now present results stemming from four numerical simulations (see table I). All runs were initialized using the random (Craya-Herring) initial conditions described in the previous section, with an initial isotropic spectrum $E(k) \sim k^{2}$ between $0<k \leq 40$ for runs $\mathrm{A}$ and $\mathrm{B}$, and $E(k) \sim k^{4}$ between $0<k \leq 40$ for runs $\mathrm{C}$ and D. Runs $\mathrm{A}$ and $\mathrm{C}$ correspond to isotropic and homogeneous turbulence (no rotation), while runs B and D are rotating, resulting in homogeneous but anisotropic flows as time evolves. The purpose of simulations $A$ and $C$ is to recover well known results for isotropic $\sim k^{2}$ and $\sim k^{4}$ turbulence, and to use them as a starting point to analyze the more complex rotating cases. Note the decay of $\sim k^{4}$ turbulence (corresponding to runs $\mathrm{C}$ and D) was studied in detail in [26], and is considered here to compare with the $\sim k^{2}$ case.

Parameters of the runs listed in table \are defined as follows: The Reynolds number is

$$
R e=\frac{l U}{\nu}
$$

where $U$ is the r.m.s velocity and the energy-containing scale $l$ is defined from the isotropic energy spectrum $E(k)$ as

$$
l=2 \pi \frac{\int E(k) k^{-1} d k}{\int E(k) d k} .
$$

The Reynolds number based on the Taylor scale $\lambda$ is

$$
R e_{\lambda}=\frac{\lambda U}{\nu}
$$




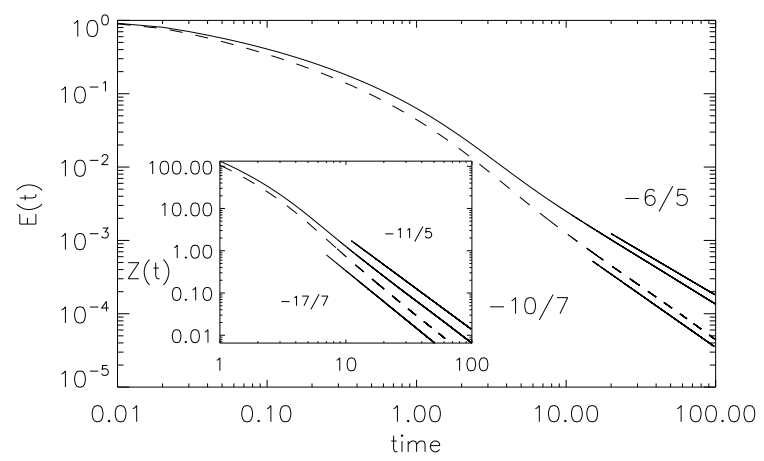

FIG. 2: Energy evolution for run A (solid) and C (dashed). Note the decay laws with exponents $\approx-6 / 5$ and $\approx-10 / 7$ respectively, after an initial transient of $\approx 10$ turn over times. Slopes are shown as a reference. Inset: enstrophy decay for the same runs. The decays approach $\approx-11 / 5$ and $\approx-17 / 7$ respectively after $t \approx 10$.

where

$$
\lambda=2 \pi\left(\frac{\int E(k) d k}{\int E(k) k^{2} d k}\right)^{1 / 2}
$$

The Rossby number is

$$
R o=\frac{U}{2 \Omega l}
$$

while the micro-Rossby number is defined as

$$
R o^{\omega}=\frac{\omega}{2 \Omega}
$$

This number should be initially of order one for the scrambling effect of waves not to completely damp the non-linear term in the Navier-Stokes equation, which would result in just an exponential decay of the energy [10].

Time-dependent parameters in table I are given for all runs at $t=0$, and also at $t=20$ (roughly ten turnover times after the self-similar decay of energy starts).

\section{A. Decay of non-rotating flows}

Figure 1 shows the evolution of the isotropic energy spectrum for run A, at times ranging from $t=0$ to $t=20$. The initial $\sim k^{2}$ law is approximately preserved over time at the smallest wavenumbers, while higher wavenumbers decay due to the effect of dissipation. This 

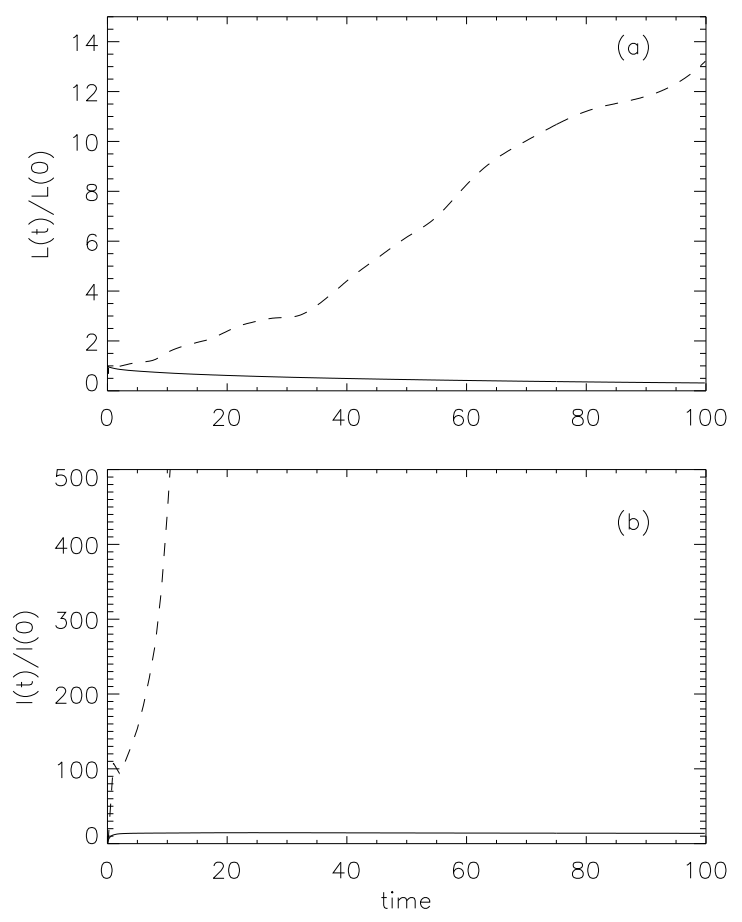

FIG. 3: (a) Evolution of the integral $L$ normalized by its initial value $L(0)$ for the isotropic run A (solid) and rotating run B (dashed). (b) Evolution of the integral $I$ normalized by its initial value $I(0)$ for the isotropic run B (solid) and rotating run D (dashed).

behavior is consistent with the quasi-conservation of $L$ in Eq. (8), for which a subsequent decay law $E \sim t^{-6 / 5}$ is expected for the energy.

Figure 2 shows the energy and enstrophy decay for runs A and C. After a transient lasting for approximately 10 turn-over times, both runs decay with different power laws. In run A, the energy decays close to $E \sim t^{-6 / 5}$ as predicted by Eq. (9), and as expected from the shape of the large-scale spectrum in Fig. 1. The enstrophy shows a faster decay close to $Z \sim t^{-11 / 5}$, consistently with phenomenological arguments which indicate that $E \sim t^{\alpha}$ leads to $Z \sim t^{\alpha-1}$ under the assumption of isotropy, as

$$
\frac{d E}{d t}=-2 \nu Z(t)
$$

The energy decay for run $\mathrm{C}$ is consistent with $E \sim t^{-10 / 7}$ as expected by Eq. (9), while the enstrophy decay is close to $Z \sim t^{-17 / 7}$, also consistent with Eq. (49). The spectrum of run $\mathrm{C}$ maintains a $\sim k^{4}$ shape for large scales (not shown). All these results are consistent with previous simulations and theoretical results [3, 27, 32]. 


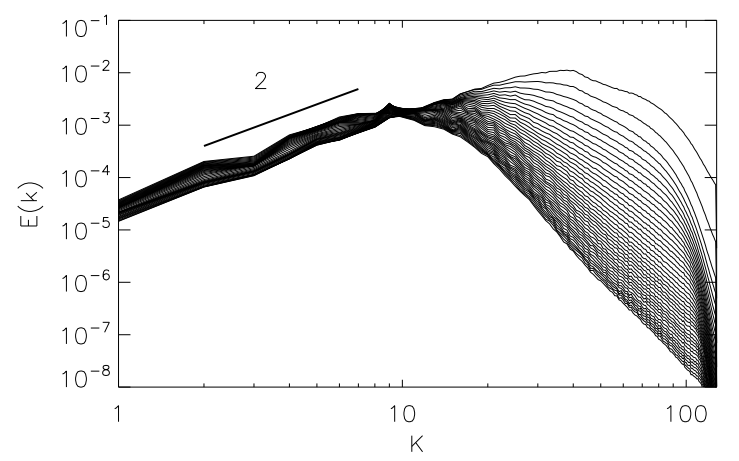

FIG. 4: Evolution of the isotropic energy spectrum $E(k)$ for run B from $t=0$ to $t=20$ with time increments $\Delta t=0.5$. Note how the initial $E \sim k^{2}$ spectrum for large scales (low wave numbers) is preserved over time, but its overall amplitude increases.

The prediction $E \sim t^{-6 / 5}$ for run A assumes $L$ remains constant during the decay. To verify this, and to further study whether $L$ also remains constant in the decay of rotating turbulence with $\sim k^{2}$ spectrum, we show the time evolution of $L$ for runs $\mathrm{A}$ and $\mathrm{B}$ in Figure 3(a). We estimated $L$ in two different ways: A fit to the spectrum with a power law $\sim k^{2}$ was computed for the smallest wavenumbers to obtain the multiplicative prefactor in Eq. (8) (proportional to $L$ if the spectrum is $\sim k^{2}$ ), as was done in [3]. We also computed the two-point correlation function from the energy spectrum using [31]

$$
\left\langle\mathbf{u} \cdot \mathbf{u}^{\prime}\right\rangle(r)=2 \int_{0}^{\infty} E(k) \frac{\sin (k r)-k r \cos (k r)}{(k r)^{3}} d k
$$

and then used Eq. (6) to compute $L$. Both methods give consistent results and in the following we show results obtained with the last method.

In run $\mathrm{A}, L$ decays to half its initial value between $t=0$ and $t \approx 10$, but after this time it only changes slowly with time and can be considered almost constant (note the energy between $t=10$ and $t=100$ changes by more than an order of magnitude). However, $L$ is far from being constant in run B; the behavior of the rotating runs is discussed in more detail in the next subsection.

Similar results were found for the evolution of $I$ in simulations $\mathrm{C}$ and D, as shown in Fig. 3(b). In run C, I grows during a short transient, but stays constant during the selfsimilar decay of the energy.

While the behavior of $L$ and $I$ in the simulations is compatible with the theoretical and phenomenological arguments discussed in Sec. III, the rapid growth of these quantities in the 


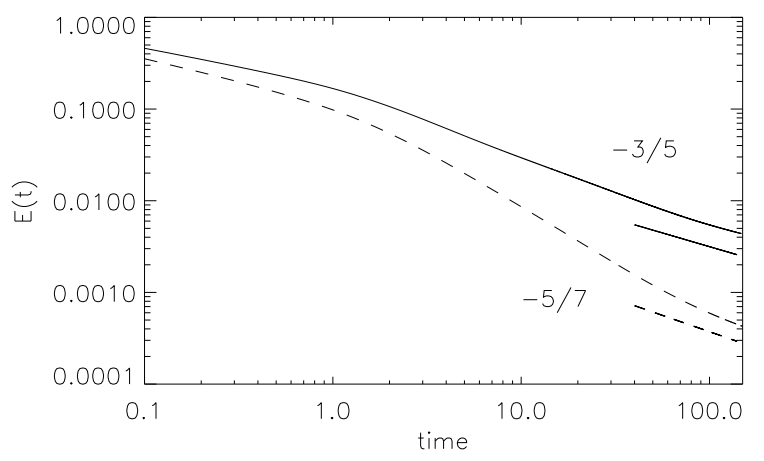

FIG. 5: Energy decay for runs B (solid) and D (dashed); $t^{-3 / 5}$ and $t^{-5 / 7}$ power laws are shown as a reference, corresponding to phenomenological predictions for Saffman and Batchelor rotating turbulence based on isotropic conserved quantities.

runs with rotation seems to question the use of isotropic integrals to derive decay laws for anisotropic flows.

\section{B. Decay of rotating flows}

The analysis for runs $\mathrm{A}$ and $\mathrm{C}$ was based on the isotropic energy spectrum and on the quasi-invariance of the isotropic integrals $L$ and $I$. However, the growth of $L(I)$ in run B (D) suggests that different arguments should be used to predict their energy decay. In this light, we now consider the anisotropic quantities introduced in Sec. II $L_{\perp}$ and $I_{\perp}$, and show that further analysis can be done by studying the evolution of anisotropic spectra and splitting the energy decay in $2 D$ and $3 D$ modes. A similar method was used in [27] to study $\sim k^{4}$ turbulence.

The evolution of the isotropic spectrum for run B is shown in Fig. 4, for different times from $t=0$ to $t=20$. As for run $\mathrm{A}$, its initial $\sim k^{2}$ behavior for large scales is preserved over time. However, its amplitude at large scales increases with time, in agreement with the evolution of $L$.

The time evolution of the total energy in run B shows that the decay is close to, but steeper than, $E \sim t^{-3 / 5}$. In Figure 5 we show the energy decay for this run (we also show the $\sim k^{4}$ case for comparison, i.e., run D). The decay deviates from the expected $\sim t^{-3 / 5}$ law derived via isotropic arguments (in the same fashion, run D deviates from the expected 


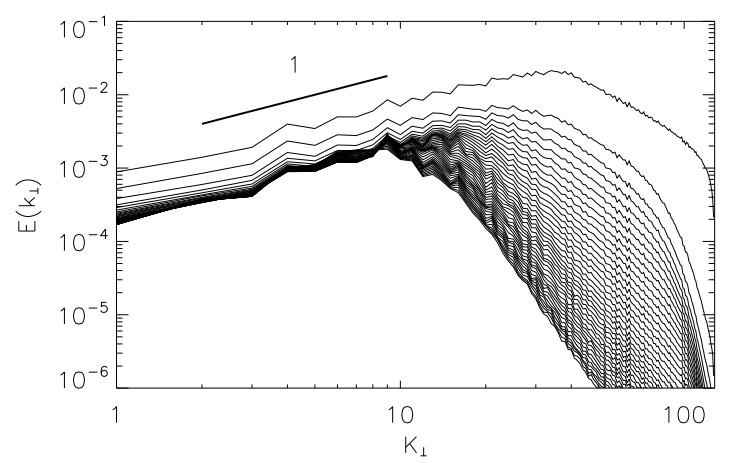

FIG. 6: Evolution of the axisymmetric energy spectrum $E\left(k_{\perp}\right)$ for run B from $t=0$ to $t=20$ with time increments $\Delta t=0.5$. The initial shape $E \sim k_{\perp}$ of spectrum at large scales (low wavenumbers) is approximately preserved over time.

$\sim t^{-5 / 7}$ law) and strictly speaking, a power law is hard to identify. Indeed, as was shown in Fig. 3, both $L$ and $I$ grow fast in runs B and D. As a result, arguments based on the quasiconservation of $L$ or $I$ should be expected to fail to predict the correct energy decay. The reason for this failure can be associated with the fact that rotating flows are not isotropic, and instead become axisymmetric with a tendency towards two-dimensionalization.

From the arguments in Sec. III, we expect only large-scale correlations in the direction perpendicular to the axis of rotation to be preserved, and either $L_{\perp}$ or $I_{\perp}$ to remain constant depending on the initial conditions. Therefore, we analyze runs B and D with the aid of the expansion in Eq. (22) and of the anisotropic energy spectrum $E\left(k_{\perp}\right)$. Following Eqs. (24) and (30), we will also separate the energy into the energy of slow modes $E_{2 D}$, and the energy of fast modes $E_{3 D}$.

In Fig. 6] we show the time evolution of $E\left(k_{\perp}\right)$ in run B. Per virtue of Eq. (42), as the isotropic spectrum is $E(k) \sim k^{2}$ for small wavenumbers, the anisotropic spectrum is $E\left(k_{\perp}\right) \sim k_{\perp}$. As for $E(k)$, the shape of the spectrum is preserved during the decay, and $E\left(k_{\perp}\right) \sim k_{\perp}$ for small $k_{\perp}$ at all times. However, unlike the isotropic spectrum, $E\left(k_{\perp}\right)$ rapidly decreases its amplitude during a short transient, and then the amplitude of the spectrum at large scales stabilizes and varies only slowly with time. Run D shows a similar behavior in $E(k)$ and $E\left(k_{\perp}\right)$, but following $E\left(k_{\perp}\right) \sim k_{\perp}^{3}$ instead.

We saw in Sec. It that constancy of $L_{\perp}\left(I_{\perp}\right)$ may be expected for $E(k) \sim k^{2}\left(\sim k^{4}\right)$ initial spectra if large-scale correlations decay fast enough, and that phenomenological analysis 

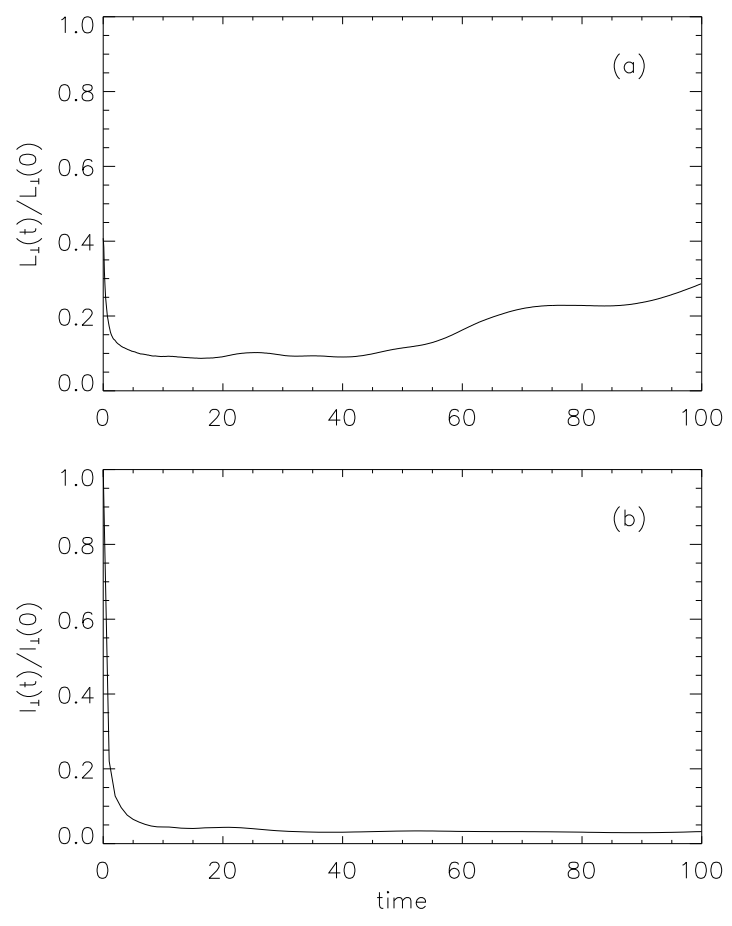

FIG. 7: (a) Ratio $L_{\perp}(t) / L_{\perp}(0)$ as a function of time for run B. (b) Evolution of $I_{\perp}(t) / I_{\perp}(0)$ in run D. Note that in both cases, these magnitudes remain approximately constant after a short initial transient.

leads then to $E_{2 D}(t) \sim t^{-1 / 2}\left(E_{2 D} \sim t^{-2 / 3}\right)$. As for $L$, we estimate $L_{\perp}$ using two different methods: By fitting the spectrum for small $k_{\perp}$, and by using Eq. (21). The two-point longitudinal correlation function for the axisymmetric case is estimated using Bessel functions and the anisotropic perpendicular spectrum (see, e.g., [26, 31]),

$$
\left\langle\mathbf{u}_{\perp} \cdot \mathbf{u}_{\perp}^{\prime}\right\rangle\left(r_{\perp}\right)=2 \int E\left(k_{\perp}\right) J_{0}\left(k_{\perp} r_{\perp}\right) d k_{\perp} .
$$

Both estimations give similar results and the curves discussed below are obtained from Eqs. (21) and (51). The same procedure was used to estimate $I_{\perp}$ from Eq. (20).

In Fig. 7 we show the evolution of $L_{\perp}$ and $I_{\perp}$ normalized by their values at $t=0$, for runs B and D respectively. Although at early times their values decrease rapidly, they remain afterwards approximately constant during the entire simulation (note that in the same simulations and at the same times, $L$ and $I$ increase by at least one order of magnitude).

The evolution of the energy for run $\mathrm{B}$ shows interesting properties (see Fig. 8); $E_{2 D}$ initially grows until $t \approx 10$, when it reaches its maximum value and begins to decrease. $E_{3 D}$ 

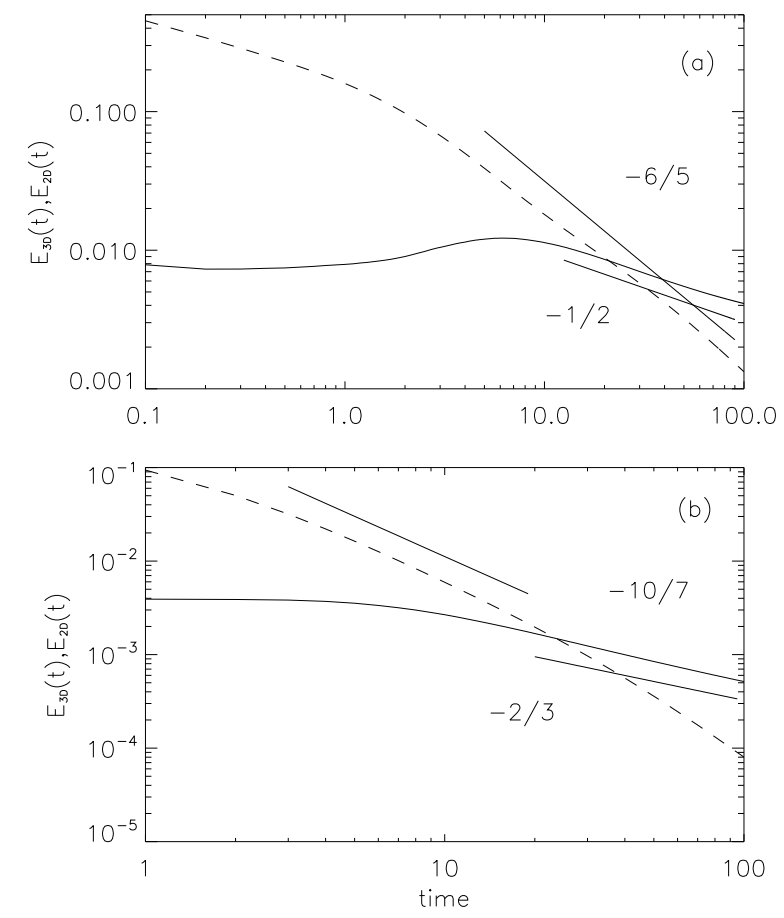

FIG. 8: (a) Time evolution of the energy $E_{3 D}$ in modes with $k_{\|} \neq 0$ (dashed), and of the energy $E_{2 D}$ in modes with $k_{\|}=0$, for run B. Power laws $t^{-6 / 5}$ and $t^{-1 / 2}$ are indicated as a reference, following phenomenological arguments respectively for the decay of the 3D energy, and for the decay of the 2D energy based on approximate constancy of $L_{\perp}$ in Saffman turbulence. (b) Same for run D. The power laws $t^{-10 / 7}$ and $t^{-2 / 3}$ correspond to the phenomenological predictions for Batchelor turbulence.

decreases faster than $E_{2 D}$ and at $t \approx 20$ both energies are comparable. After that time, the system is dominated by the energy in the slow modes. Starting at $t \approx 10$, both energies show a decay compatible with power laws with different exponents. $E_{2 D}(t)$ decays close to $\sim t^{-1 / 2}$, in agreement with Eq. (30) and with the approximate constancy of $L_{\perp}$, while $E_{3 D}$ decays close to $\sim t^{-6 / 5}$, the value expected from Eq. (9) for the decay of three-dimensional Saffman turbulence.

A distinct decay of $E_{2 D}$ and $E_{3 D}$ is also observed in run D (corresponding to $\sim k^{4}$ turbulence, also shown in Fig. 8). The decay of the energy in slow modes is compatible with $E_{2 D}(t) \sim t^{-2 / 3}$, as expected for Batchelor turbulence from Eq. (30) and with the approximate constancy of $I_{\perp}$ shown in Fig. 7. The 3D energy shows a decay that is close to $E_{3 D} \sim t^{-10 / 7}$. 


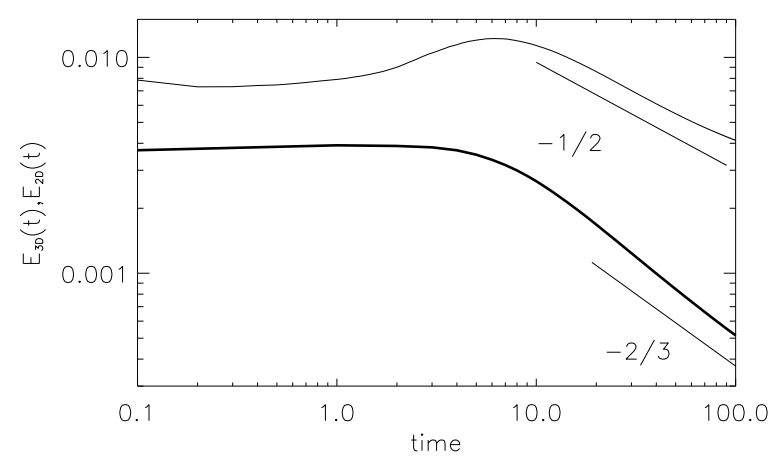

FIG. 9: $E_{2 D}$ decay as a function of time for runs B (thin) and D (thick). The expected decays based on constancy of $L_{\perp}$ and $I_{\perp}$ are indicated as a reference. The agreement in this case is much better than when assuming constancy of the isotropic integrals $L$ and $I$ (see Fig. 5 ).

The decay of $E_{2 D}$ in runs B and D is compared in Fig. 9, The two different decay laws followed by the energy in the two runs can be clearly identified. The result indicates that the decay of rotating turbulence is affected by large scale correlations, as different power laws can be observed for $E(k) \sim k^{2}$ and $\sim k^{4}$ initial spectra.

The different power laws followed by $E_{2 D}$ and $E_{3 D}$ after $t \approx 10$ in both runs point to a negligible interchange of energy between slow and fast modes at late times, as required for an equation like Eq. (24) to hold. Runs B and D have initial values of the Rossby number which correspond to the intermediate Rossby range studied in [25]. In this range, maximal energy transfer from $3 \mathrm{D}$ to $2 \mathrm{D}$ modes takes place at early times, with $E_{2 D}$ in some cases growing from energy in 3D modes as a result (see Fig. 8 (a)). After this phase, the independent decay of the two energies implies that these exchanges are small compared with, e.g., the transfer of energy from vortical motions at large scales to vortical motions at smaller scales in $E_{2 D}$, and the transfer from $3 \mathrm{D}$ modes to $3 \mathrm{D}$ modes in $E_{3 D}$.

\section{Energy exchange between $2 \mathrm{D}$ and $3 \mathrm{D}$ modes}

A measure of the exchange of energy between 2D and 3D modes per unit of time can be obtained from the flux of energy across planes in Fourier space with normal $k_{z}$. The amount 

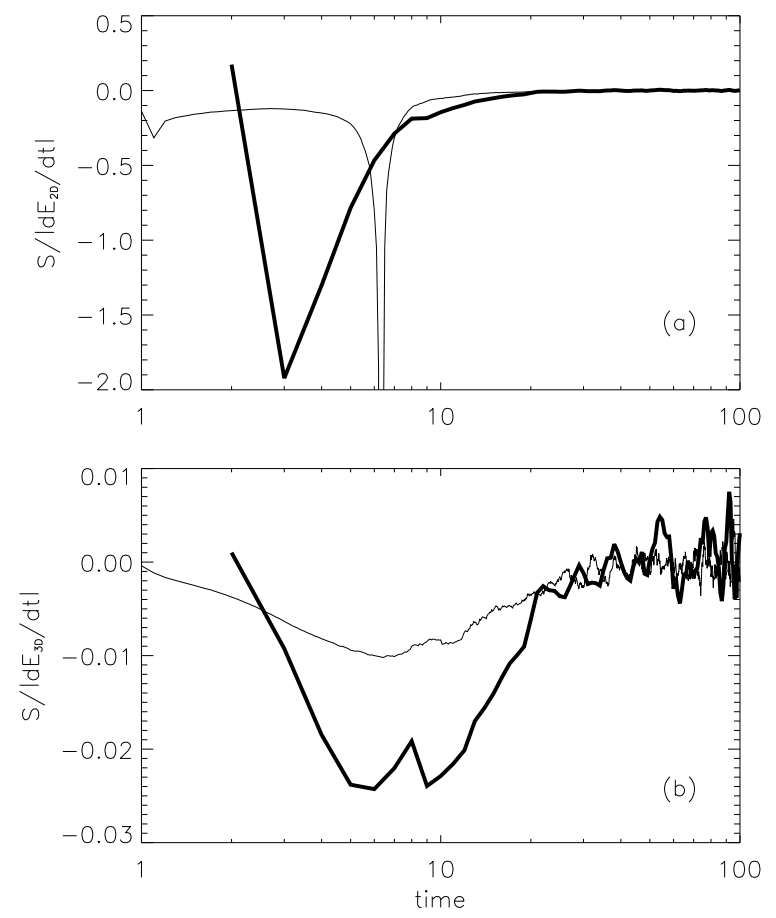

FIG. 10: (a) Energy interchanged per unit of time $S$ between 2D and 3D modes, (a) normalized by $\left|d E_{2 D} / d t\right|$, and (b) normalized by $\left|d E_{3 D} / d t\right|$, for runs $\mathrm{B}$ (thin) and $\mathrm{D}$ (thick). Positive values of $\mathrm{S}$ represent flux of energy from $2 \mathrm{D}$ to $3 \mathrm{D}$ modes, while negative values indicate flux from 3D to 2D motions. At early times $S$ is negative, indicating energy is transferred from 3D to 2D modes. After $t \approx 10$ in run $\mathrm{B}$, and $t \approx 10$ in run $\mathrm{D}$, when the distinct power law decays are observed for $E_{2 D}(t)$ and $E_{3 D}(t)$ (Fig. 8), $S$ becomes negligible.

of energy per unit of time going through any of such planes is given by

$$
\Pi\left(k_{\|}\right)=-\iiint_{k_{\|}=0}^{k_{\|}} \hat{\mathbf{u}}_{\mathbf{k}}^{*} \cdot(\widehat{\mathbf{u} \cdot \nabla \mathbf{u}})_{\mathbf{k}} d k_{x} d k_{y} d k_{z},
$$

where the integrals in $k_{x}$ and $k_{y}$ run over their entire range (see [47] for definitions of anisotropic fluxes and spectral transfer functions), and the hat denotes Fourier transform. The energy transferred from 2D modes to 3D modes per unit of time is then

$$
S=\Pi\left(k_{\|}=0\right)
$$

When $\mathrm{S}$ is positive, it represents transfer of energy from 2D to 3D motions (i.e., energy going from $k_{\|}=0$ towards larger values of $k_{\|}$, resulting in an effective source of 3D energy coming from 2D loses), while when negative it represents transfer from 3D to 2D motions (i.e., a 

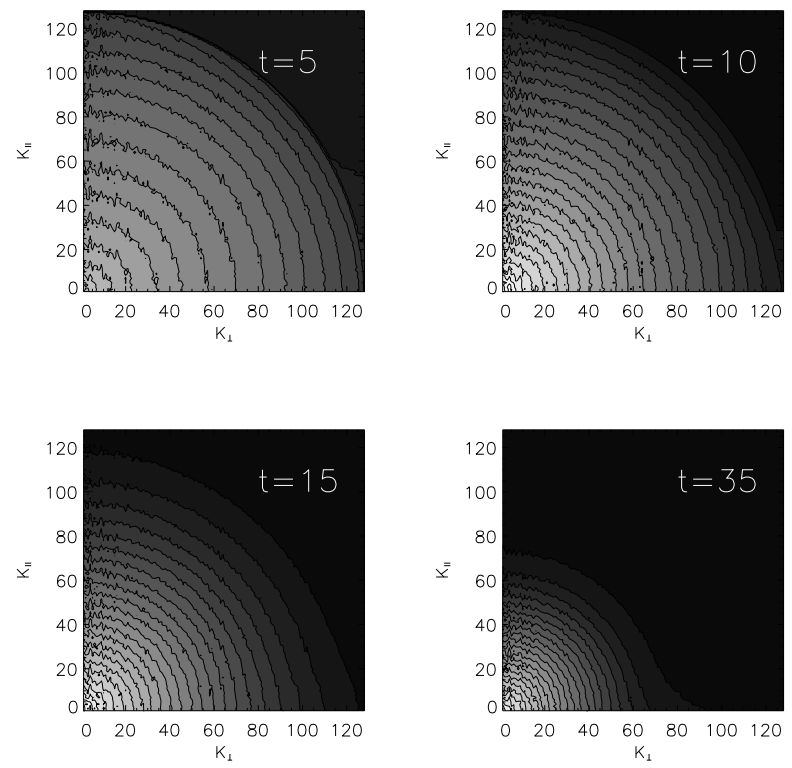

FIG. 11: Axisymmetric energy spectrum $e\left(k_{\|}, k_{\perp}\right) / \sin \theta$ at different times in run A. The circular contour levels indicate an isotropic energy distribution.

source of 2D energy from 3D loses). For these sources (or loses) of energy to be negligible, they must be small when compared with the time derivatives $d E_{2 D} / d t$ and $d E_{3 D} / d t$. When they are, the decay of $E_{2 D}$ and of $E_{3 D}$ can be considered independently.

Figure 10 shows the function $S$ normalized by $\left|d E_{2 D} / d t\right|$ and $\left|d E_{3 D} / d t\right|$ for runs B and D. In both runs $S$ is negative before $t \approx 10$, indicating energy goes from 3D to 2D modes, and explaining the growth of $E_{2 D}$ at early times in run B. Note that in run B, $\left|d E_{2 D} / d t\right|$ becomes zero at $t \approx 5$, resulting in an infinite value of the ratio $S /\left|d E_{2 D} / d t\right|$ in Fig. 10 (a). Later, for $t \approx 10$ in run $\mathrm{B}$, and for $t \approx 20$ in run $\mathrm{C}, S /\left|d E_{2 D} / d t\right|$ and $S /\left|d E_{3 D} / d t\right|$ become small, indicating the interchange of energy between slow and fast modes becomes negligible when compared with the energy decay rates, and justifying the use of separate balance equations to study the decay of $E_{2 D}$ and $E_{3 D}$. Indeed, to have $S \ll\left|d E_{2 D} / d t\right|$ and $S \ll\left|d E_{3 D} / d t\right|$ is enough to consider equations for the evolution of $E_{2 D}$ and $E_{3 D}$ as was done in Sec. III, as is a more precise definition of what was meant in that section by the condition of "weakly coupled" 2D and 3D modes.

It is worth pointing out that the fact that $S /\left|d E_{2 D} / d t\right|$ and $S /\left|d E_{3 D} / d t\right|$ are much smaller than unity does not imply that the 2D and 3D modes are completely decoupled, and only implies that almost no energy is interchanged between slow and fast modes during the self- 

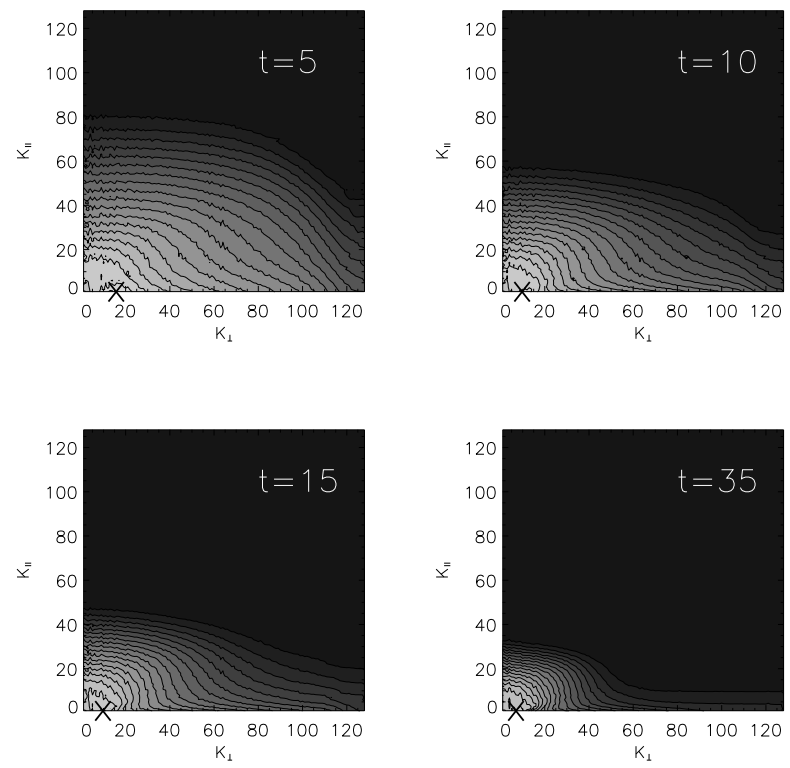

FIG. 12: Axisymmetric energy spectrum $e\left(k_{\|}, k_{\perp}\right) / \sin \theta$ at different times in run B. For rotating flows, the spectral distribution of energy becomes anisotropic with more energy near the $k_{\|}=0$ plane. The cross indicates the maximum of the spectrum at the different times.

similar decay. Triadic interactions, e.g., between two fast modes and one slow mode, can still occur and be relevant, as long as the slow mode is only an intermediary and does not receive from nor give net energy to the fast modes. Indeed, such interactions between slow and fast modes that preserve the 2D and 3D energy separatedly would be required to have $d E_{2 D} / d t$ proportional to $1 / \Omega$ in Eq. (25), as in their absence the decay of $E_{2 D}$ should be that of 2D Navier-Stokes and independent of $\Omega$ (note triadic interactions between three slow modes are trivially resonant and independent of $\Omega$ ).

\section{Energy spectral distribution}

A deeper understanding of the development of anisotropy in the flow cannot be obtained solely from studying the reduced spectrum $E\left(k_{\perp}\right)$ and the isotropic spectrum $E(k)$. To further investigate the energy spectral distribution, we present contour plots of the axisymmetric energy spectrum $e\left(k_{\|}, k_{\perp}\right)$ for runs A, B, and D in Figs. 11, 12, and 13 respectively. Note that in order to obtain circular contour levels when the spectral distribution of the energy is isotropic, in these figures the axisymmetric energy spectrum is divided by $\sin \theta$, 

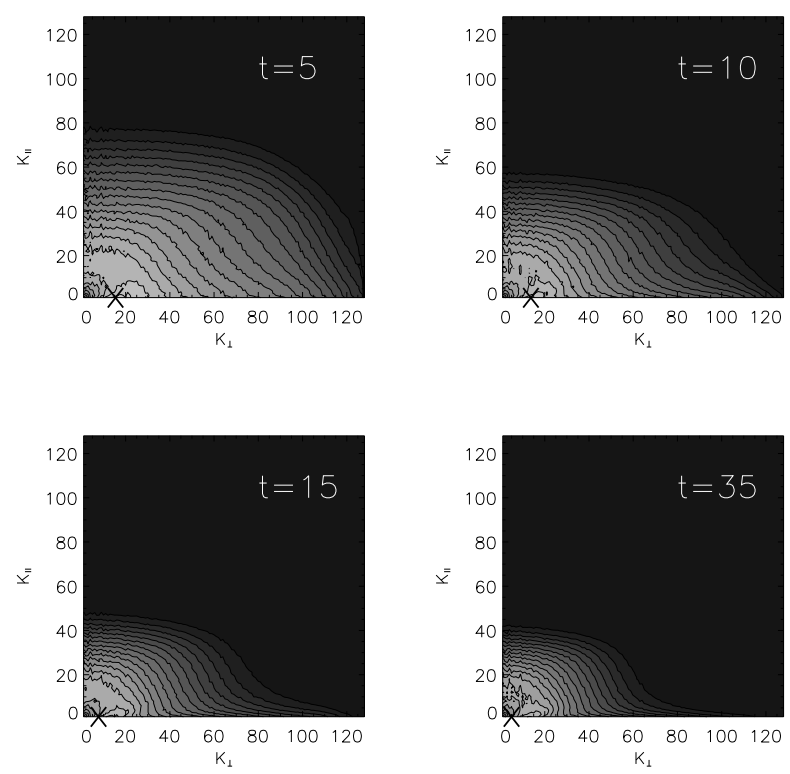

FIG. 13: Axisymmetric energy spectrum $e\left(k_{\|}, k_{\perp}\right) / \sin \theta$ at different times in run D. As in run B, the spectral energy distribution is anisotropic. The cross indicates the maximum of the spectrum at the different times.

where $\theta=\arctan \left(k_{\|} / k_{\perp}\right)$.

In the absence of rotation (Fig. 11) the spectrum shows an isotropic energy distribution evidenced by circular contour levels which maintain their shape as the flow decays. However, when rotation is present, the spectral distribution of the energy becomes anisotropic with more energy near the $k_{\|}=0$ axis (see Figs. 12 and 13). Already at $t=10$, the maximum of the axisymmetric energy spectrum takes place in the $k_{\|}=0$ axis, in agreement with the preferential transfer from 3D modes towards slow 2D modes observed at early times in the flux in Fig. 10 (as a matter of fact, the maximum of the spectrum is already located in the $k_{\|}=0$ axis for times as early as $\left.t=5\right)$.

After $t=10$, the exchange of energy between 2D and 3D modes becomes negligible. As already observed in the time evolution of $E_{2 D}$ and $E_{3 D}$, the energy in the 3D modes decays faster than the energy in $2 \mathrm{D}$ modes. This results in an increase of the spectral anisotropy as time evolves, with most of the energy near the $k_{\|}=0$ axis at late times. At the same time, the peak of the spectrum slowly moves through the $k_{\|}=0$ axis towards smaller values of $k_{\perp}$, although even at late times the peak is sufficiently far from $k_{\perp}=1$.

The shape of the spectrum near the $k_{\|}=0$ plane is of interest for many theories of 

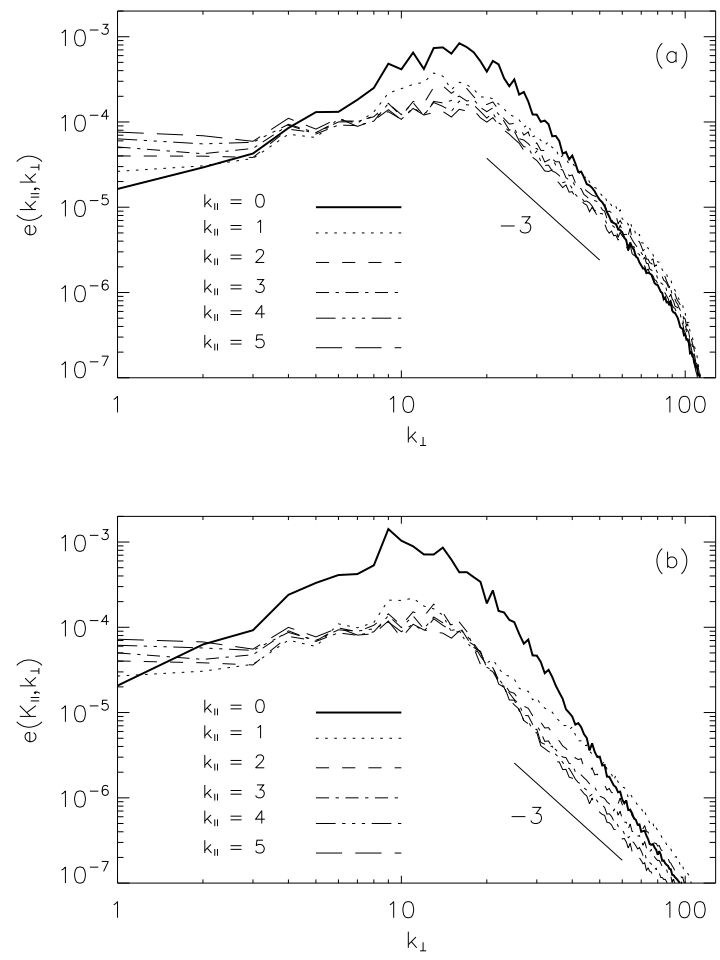

FIG. 14: Axysimmetric energy spectrum $e\left(k_{\|}, k_{\perp}\right)$ for run B for different values of $k_{\|}$at (a) $t=5$, and (b) $t=10 . \mathrm{A} k_{\perp}^{-3}$ slope is shown as a reference.

homogeneous rotating turbulence (see, e.g., [11, 48]). It is important to note that here the domain with finite size results in a discrete set of values for $k_{\|}$so the $k_{\|} \rightarrow 0$ limit cannot be studied, unlike homogeneous flows in infinite domains for which $k_{\|}$is a continuum. Bearing in mind this limitation, we show in Figs. 14 and 15 the axisymmetric energy spectrum $e\left(k_{\|}, k_{\perp}\right)$ for fixed values of $k_{\|}$(from 0 to 5 ), for runs $\mathrm{B}$ and $\mathrm{D}$ at two different times $(t=5$ and $t=10)$.

Figures 14 and 15 confirm that most of the energy is contained in modes with $k_{\|}=0(2 \mathrm{D}$ modes), and that the wavenumber $k_{\perp}$ at which the peak of the spectrum takes place slowly decreases with time (although the wavenumber is larger than $k_{\perp}=1$ ). As an example, in run $\mathrm{B}$ the peak of the spectrum for $k_{\|}=0$ is at $k_{\perp} \approx 20$ at $t=5$, at $k_{\perp} \approx 15$ at $t=10$, and at $k_{\perp} \approx 8$ at $t=35$. The spectrum for modes with $k_{\|}=0$ also shows a positive slope for wavenumbers smaller than the energy containing wavenumber, and the slope seems to be approximately preserved as the system evolves in time.

The spectra $e\left(k_{\|}, k_{\perp}\right)$ for $k_{\|} \geq 1$ are approximately flat for small wavenumbers (specially 

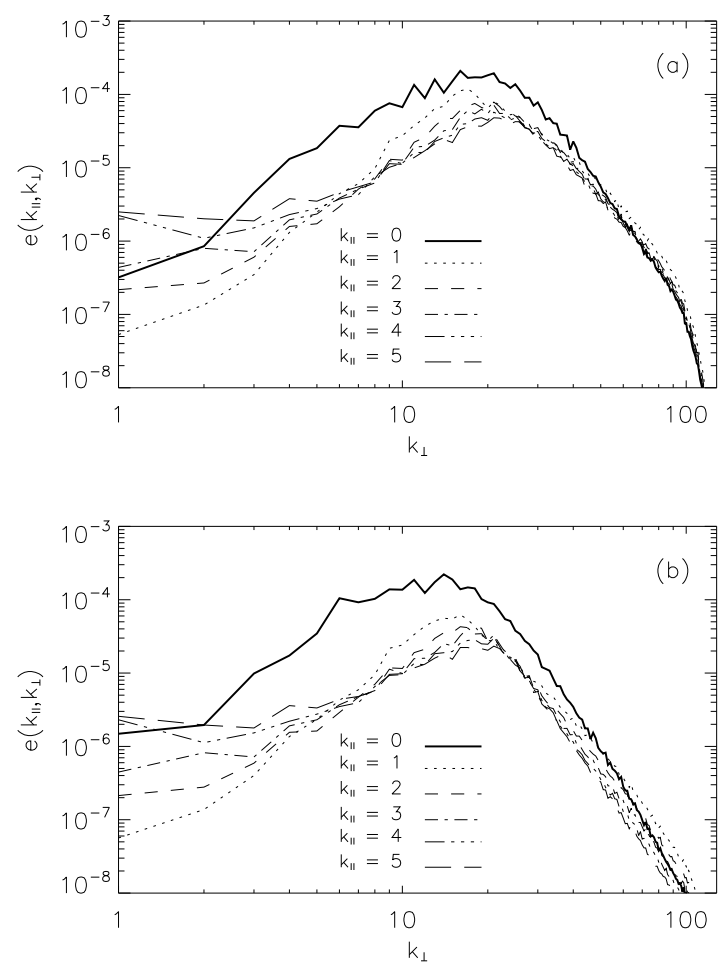

FIG. 15: Axysimmetric energy spectra $e\left(k_{\|}, k_{\perp}\right)$ for run D for different values of $k_{\|}$, at (a) $t=5$, and (b) $t=10$.

for run B), and show a slope compatible with a $k_{\perp}^{-3}$ power law (specially for early times, at $t=5$, and for the spectrum corresponding to $k_{\|}=1$ ). This is consistent with the scaling $e\left(k_{\|}, k_{\perp}\right) \sim k_{\|}^{-1 / 2} k_{\perp}^{-3}$ valid for $k_{\|} / k_{\perp} \ll 1$ derived from inertial wave turbulence equations in [48], and also observed before in numerical simulations in [30].

\section{CONCLUSIONS}

We have analyzed numerical simulations of freely decaying rotating turbulence in periodic domains in the regime of intermediate Rossby number $\left(R_{o} \approx 0.1\right)$, with initial energy spectra $\sim k^{2}$ and $\sim k^{4}$. We first presented a brief theoretical discussion of conserved integral quantities for rotating turbulence using a von Kármán-Howarth equation which includes the Coriolis term due to rotation, extending previously derived results for Saffman turbulence [12], considering also the Batchelor spectrum. Assuming 2D and 3D modes are only weakly coupled in rotating turbulence, constancy of the anisotropic integral quantities $L_{\perp}$ or $I_{\perp}$ was 
used to derive phenomenologically the energy decay rate expected for energy in 2D modes.

In simulations without rotation, we recovered well known results for isotropic $\sim k^{2}$ and $\sim k^{4}$ turbulence, and also verified the approximate constancy of the isotropic integrals $L$ and $I$. On the other hand, in the simulations with rotation a self-similar decay was difficult to identify for the total energy, while $L$ and $I$ were found to grow rapidly during the entire decay. However, the decay of energy in two-dimensional and three-dimensional modes followed distinct power laws, in agreement with the phenomenological predictions and with constancy of $L_{\perp}$ and $I_{\perp}$, which were also observed to remain approximately constant in the simulations.

The separate power laws followed by $E_{2 D}(t)$ and $E_{3 D}(t)$ in decaying rotating turbulence require the interchange of energy between slow and fast modes to be negligible. We verified that this was the case by studying the flux of energy across planes with normal $k_{z}$ in Fourier space. The results showed that energy is initially transferred from 3D to $2 \mathrm{D}$ modes, as expected for rotating turbulence at intermediate Rossby number [25], but that later the flux of energy between 3D and 2D modes becomes negligible. Contour levels of the axisymmetric energy spectral distribution show that in presence of rotation the energy accumulates rapidly near the $k_{\|}=0$ plane, and that at later times the anisotropy increases as a result of the different decay rates of the $E_{2 D}$ and $E_{3 D}$ components of the total energy.

Altogether, the results show that rotating turbulence is indeed affected by initial large

scale correlations in the flow, as different decay laws arise for $\sim k^{2}$ and $\sim k^{4}$ initial spectra.

\section{Acknowledgments}

The authors acknowledge support from UBACYT Grant No. 20020090200692, PICT Grant No. 2007-02211, and PIP Grant No. 11220090100825.

[1] W.K. George, Phys. Fluids A 4, 1492 (1992).

[2] P.A. Krogstad and P.A. Davidson, J. Fluid Mech. 10, 418 (2011).

[3] T. Ishida, P. A. Davidson, and Y. Kaneda, J. Fluid Mech. 564, 455 (2006).

[4] G.K. Batchelor and I. Proudman, Phil. Trans. R. Soc. Lond. A 248, 369 (1956).

[5] P.G. Saffman, J. Fluid Mech. 27, 581 (1967). 
[6] P.G. Saffman, Phys. Fluids 10, 1349 (1967).

[7] P.A. Davidson, J. Fluid Mech. 632, 329 (2009).

[8] L.D. Landau and E.M. Lifshitz, 1st edn. Pergamon, (1959).

[9] C. Cambon and L. Jacquin, J. Fluid Mech. 202, 295 (1989).

[10] C. Cambon, N.N. Mansour, and F.S. Godeferd, J. Fluid Mech. 337, 303 (1997).

[11] F. Bellet, F.S. Godeferd, J.F. Scott, and C. Cambon, J. Fluid Mech. 562, 83 (2006).

[12] P.A. Davidson, J. Fluid Mech. 663, 268 (2010).

[13] N. Okamoto, P.A. Davidson, and Y. Kaneda, J. Fluid Mech. 651, 295 (2010).

[14] L.J.A. van Bokhoven, H.J.H. Clercx, G.J.F. van Heijst, and R.R. Trieling, Phys. Fluids 21, 096601 (2009).

[15] C. Lamriben, P-P. Cortet, and F. Moisy, Phys. Rev. Lett. 107, 024503 (2011).

[16] P.J. Staplehurst, P.A. Davidson, and S.B. DalEziel, J. Fluid Mech. 598, 81 (2008).

[17] C.N. Baroud, B.B. Plapp, H.L. Swinney, and Zhen-Su She, Phys. Fluids 15, 2091 (2003).

[18] C. Morize and F. Moisy, Phys. Fluids 18, 065107 (2006).

[19] F. Moisy, C. Morize, M. Rabaud, and J. Sommeria, J. Fluid Mech. 666, 5 (2011).

[20] L. Jacquin, O. Leuchter, C. Cambon, and J. Mathieu, J. Fluid Mech. 220, 1 (1990).

[21] X. Yang and J. A. Domaradzki, Phys. Fluids 16, 4088 (2004).

[22] W.C. Müller and M. Thiele, Europhys. Lett. 77, 34003 (2007).

[23] L. J. A. van Bokhoven, C. Cambon, L. Liechtenstein, F. S. Godeferd, and H. J. H. Clercx, J. of Turbulence 9, 6 (2008).

[24] T. Teitelbaum and P.D. Mininni, Phys. Rev. Lett. 103, 014501 (2009).

[25] L. Bourouiba and P. Bartello, J. Fluid Mech. 587, 161 (2007).

[26] T. Teitelbaum and P.D. Mininni, Phys. Scr. T142, 014003 (2010).

[27] T. Teitelbaum and P.D. Mininni, Phys. Fluids 23, 065105 (2011).

[28] K.D. Squires, J.R. Chasnov, N.N. Mansour, and C. Cambon, Proceedings of the 74th fluid dynamics symposium on application of direct and large eddy simulation to transition and turbulence, (Chania, Crete, Greece), AGARD-CP-551 (1994).

[29] L. Bourouiba, D.N. Straub, and M.L. Waite, J. Fluid Mech. 690, 129 (2012).

[30] M. Thiele and W.C Mller, J. Fluid Mech. 637, 425 (2009).

[31] P.A. Davidson, Turbulence: An Introduction for Scientists and Engineers. Oxford: Oxford Univ. Press (2004). 
[32] A.N. Kolmogorov, Dokl. Akad. Nauk SSSR 31, 538 (1941).

[33] P.S. Iroshnikov, Sov. Astron. 7, 566 (1963).

[34] R.H. Kraichnan, Phys. Fluids 8, 1385 (1965).

[35] P.D. Mininni, A. Alexakis, and A. Pouquet, Phys. Fluids 21, 015108 (2009).

[36] S. Fox and P.A. Davidson, Phys. Fluids 21, 125102 (2009).

[37] L.M. Smith and Y. Lee, J. Fluid Mech. 535, 111 (2005).

[38] J. Baerenzung, H. Politano, Y. Ponty,and A. Pouquet, Phys. Rev. E 77, 046303 (2008).

[39] J. Baerenzung, P.D. Mininni, A. Pouquet, H. Politano, and Y. Ponty, Phys. Fluids 22, 025104 (2010).

[40] J. Baerenzung, D. Rosenberg, P.D. Mininni, A.Pouquet, J. Atmos. Sci. 68, 2757 (2011).

[41] D.O. Gómez, P.D. Mininni, and P. Dmitruk, Adv. Sp. Res. 35, 899 (2005).

[42] A. Craya, P. S. T.Ministère de l'Air 345, (1958).

[43] J.R. Herring, Phys. Fluids 17, 859 (1974).

[44] J. Lee, Phys. Fluids 22, 40 (1979).

[45] C. Cambon, C. Tesseidre, and D. Jeandel, J. Mec. Theor. Appl. 4, 629 (1985).

[46] P.D. Mininni, D. Rosenberg, and A. Pouquet, J. Fluid Mech. 699, 263 (2012).

[47] P.D. Mininni, Annual Rev. Fluid Mech. 43, 377 (2011).

[48] C. Cambon, R. Rubinstein, and F. Godeferd, New J. Physics 6, 73 (2004). 